\title{
ELECTRIC VEHICLE BASED ON STANDARD INDUSTRIAL COMPONENTS
}

\author{
José Fernández Ramos ${ }^{1}$ and Foroohar Aghili Khatir ${ }^{2}$ \\ ${ }^{1}$ Departamento de Electrónica \\ Universidad de Málaga \\ Complejo Tecnológico de Teatinos. 2.2.39 - Boulevar Louis Pasteur, $n^{\circ}$ 35, Cod. 29071 MÁLAGA (Spain) \\ Phone/Fax number:+0034 952131441 / +0034 952 133324, e-mail: josefer@ctima.uma.es \\ ${ }^{2}$ Asociación para la promoción de los vehículos eléctricos y no contaminantes de España (AVELE) \\ Apartado de correos 265. Torremolinos - Málaga (Spain) \\ Phone: +0034 952 389370, e-mail: info@avele.org
}

\begin{abstract}
The aim of this paper is to presents the complete design of an electric vehicle by using standard industrial components as VRLA batteries, AC induction motors and standard frequency converters.

In comparison with dedicated components, the use of standard components has the following advantages: higher reliability, low price, broad range of products and suppliers, and high availability and technological independence. Besides this, we show that these components allow to make an easy and efficient electric differential without speed or steering sensors and they can perform regenerative braking.
\end{abstract}

\section{Key words}

Electric vehicle, electric differential, standard frequency converter, AC induction motor.

\section{Introduction}

The electric vehicle (EV) has a long history, dating back to 1839 when the first EV was built in Scotland. In 1900, worldwide there were more than 50 factories of EVs, which produced more than 4000 units per year. In 1908, there was 34000 EVs of Buffalo Corporation in the streets of USA, French and United Kingdom . From 1912, because to help of Petrol companies, internal combustion vehicles (ICVS) began to gain popularity and production of electric vehicles declined until it stopped completely. [1]. Currently, the EV is again considered a viable alternative to the ICV due to several factors, including the progressive rising prices of fossil fuels, its tremendous impact in increasing air pollution and carbon dioxide emissions and the great step forward that have experienced battery technologies and power electronic control.

Today there are three basic types of EVs depending on the source of electrical energy they use: the original concept or Battery EV (BEV), powered by rechargeable batteries (usually plug-in EVs), the hybrid EV (HEV), powered by batteries and an internal combustion engine and the Fuell Cell EV (FCEV), powered by hydrogen, ethanol or other type of fuel cell [2].

We believe that BEVs are the most promising means to improve the near-term sustainability of the short range transportation because BEVs do not need any fuel. This is very important in remote areas, where it is difficult to establish fuel stations but it is possible to put photovoltaic or wind generators to recharge batteries. In addition, vehicle-to-grid (V2G) power technology allows that plug-in BEVs can also be used to store excess electrical energy (eg. generated by renewable energy sources), which can be returned to the grid when needed [3].

The initial concept of BEV, which has three basic components: rechargeable battery, power converter and electric motor, is still in force although today there are many more options to choose each component. There are many different types of batteries, such as VRLA, Ni-Cd, $\mathrm{Ni}-\mathrm{MH}, \mathrm{Na} / \mathrm{NiCl} 2$, Li-ion or Li-polymer, among others; different topologies of power converters (choppers, inverter, PWM, resonant) and control schemes (variable voltage and frequency, vector, neural, fuzzy, etc.) and also a great variety of engine types (permanent magnet DC, series DC, separately excited DC, brushless DC, AC induction, switched reluctance ...). [2]

The key design of the BEV depends on how to choose each of these three components. The selection criteria can be very diverse: maximize efficiency, reliability, simplicity of control, number of recharges (battery life cycle), or minimize weight, recharge time or energy consumption. This choice is also conditioned by the performance that should have the vehicle, such as maximum speed, range, number of passengers, power, etc.

This paper deals with the complete design of an electric vehicle based on components that have been selected on the basis of choosing the subsystems more standardized and widely used in industry. This methodology has been used successfully in other fields of renewable energies as 
photovoltaic pumping systems [4]- [5]. The advantages over other possible choices are:

- High reliability. They are highly tested components which have demonstrated an optimal performance in all types of adverse conditions in the industry.

- $\quad$ Low price, because its production is very high and they have very different applications.

Broad range of products and suppliers.

Availability of components virtually anywhere in the world. These components can easily be purchased or repaired in the majority of countries, providing an important technological independence that can enable geographic extent of these vehicles.

\section{Selection of components for the BEV}

a) Selection of batteries.

Some of the major features that should have traction batteries for EVs are as follows [7]:

- High-energy density can be attained with one charge to provide a long range or mileage

- The high-energy density makes it possible to attain stable power with deep discharge characteristics to allow for acceleration and ascending power capability of the $\mathrm{EV}$

- Long cycle life with maintenance free.

- High mechanical and electrical robustness

- Wide acceptance as a recyclable battery from the environmental standpoint

- Low cost and readily available in the market

None of the batteries today available has all these characteristics, therefore, the choice of a type of battery depends on the characteristic that is considered the most important in the design.

We can consider three main types of batteries suitable for EV traction: Valve regulated lead-acid (VRLA), NiMh and solid state Li-ion.

VRLA batteries are a maintenance-free evolution of the classic flooded lead-acid battery. Its energy density and life cycle is lower than the NiMH and Li-ion batteries, but instead, are cheaper and are readily available in highpower formats.

Since its introduction in the early and mid 1980 the $\mathrm{NiMH}$ rechargeable batteries have reached a very important market share in the field of rechargeable batteries of low voltage-low power and general purpose (type AAA, AA, C, D, 9V, etc.) and its energy density has improved considerably. They also are manufactured in large-scale formats (eg used in the Toyota Prius II) although its market is much more restricted.

The solid state Li-ion batteries now monopolize the entire market for laptops and cell phones. Its technology is mature and have some characteristics that make it best suited to power BEVs: High energy densities, long life cycle and good pulse power density. However, today are still quite expensive and difficult to acquire in small amounts in high-power formats.

In our design, we have prioritized the aspects of low cost and easy availability in the market, so we selected VRLA batteries.

b) Selection of Motor and power converter.

The major requirements of the EV motor drive are summarized as follows [2]:

1) High instant power and high power density.

2) High torque at low speeds for starting and climbing, as well as high power at high speed for cruising.

3) Very wide speed range including constant-torque and constant-power regions.

4) Fast torque response.

5) High efficiency over wide speed and torque ranges.

6) High efficiency for regenerative braking.

7) High reliability and robustness for various vehicle operating conditions.

8) Reasonable cost and easy availability on the market.

As with batteries, there is no electric motor that meets all these conditions, so it is necessary to establish which of these are priority in our selection.

Several types of electric motors have been used for traction applications. DC series wound motors are easy to control and they have a high starting torque, a very compact design and it can run at high speed, so they have been extensively used in railways and early BEVs. However, in order to propel the modern BEVs, they present two major drawbacks: They are less efficient than other types of engines and they can not be used for regenerative braking.

A modern version of DC shunt wound motor, the separately excited DC motor is used in little BEVs as golf cars. Advanced electronic control systems make possible a precise control of torque and speed of these motors and they can implement regenerative braking. Its low operating voltage makes their use is restricted to lowpowered vehicles. Moreover, as DC series wound motors, these motors have brushes, hence, they require regular maintenance.

The induction asynchronous motor (AC motor) is robust, cheap, efficient, and widely used in the industry around the World. Their main problem for use in batterypowered EVs was that they require a three-phase AC power supply. This problem has disappeared with the development of variable speed drives or frequency converters. These devices enable precise control of the rotating frequency and the motor torque. In addition, they include other great features as analog and digital inputs and outputs, PID control and can be supplied with direct current. Thus, the association of AC motors and frequency converters is very appropiated to propel EVs. 
The most promising type of engine for use in EVs is the PM brushless. This motor has more power density and efficiency than the AC motor, but has the disadvantages of being more expensive and complex, and it is not produced in high scale as $\mathrm{AC}$ motor.

Therefore, we have selected the AC motor and standard frequency converter (SFC) for designing our BEV.

\section{BEV configuration.}

Compared with a ICVS, the power train configuration of an EV is rather flexible. There are two fundamental differences. One is that the electric motor has a constant torque characteristic in a wide range of speeds from zero. This makes it unnecessary the gearbox. The other difference is that the EV can be equipped with more than one electric motor, making it unnecessary the use of a mechanical differential.

The simplest configuration that uses these advantages consists in two electric motors coupled by gears to rear wheels and controlled with two independent SFCs that perform an electric differential (ED). For simplicity, the front wheels are not used for traction, they are used for steering.

The ED is characterized by the following features [8]:

1) There is no mechanical link between the drive wheels.

2) The traction power is separately applied to each wheel by the speed controller.

3) In a turn, the speed controller will apply less power to the inner wheel.

4) The ED simulates a differential lock while the front wheels are driving straight paths.

The kinematic model of the car when tracking a curve path is given by the Ackerman equations [8]. Such equations describe the relationship between the car angular velocity $\omega$ and the inner $\left(\omega_{2}\right)$ and outer $\left(\omega_{1}\right)$ wheel velocities, assuming that the vehicle speed $(v)$ is constant when cornering, the steering angles of the two front wheels are equivalent and there are no slip on the tires.

If $R$ is the turn radius, $L$ the wheelbase, $D$ the distance between wheels of the same axle, $\delta$ the steering angle and $r$ the wheel radius, the equations are:

$\omega_{1}=\frac{\omega}{r} \cdot\left(R+\frac{D}{2}\right) ; \quad \omega_{2}=\frac{\omega}{r} \cdot\left(R-\frac{D}{2}\right) ;$

$\tan \delta=\frac{L}{R} ; \quad v=\omega \cdot R$

No slipping occurs, so the total torque must equally be distributed on the two rear wheels: $T_{1}=T_{2}=T$. The power developed by each motor and the total power are the following:

$P_{1}=\omega_{1} \cdot T ; \quad P_{2}=\omega_{2} \cdot T ;$
$P_{\text {TOT }}=2 \cdot \frac{\omega}{r} \cdot T \cdot R=2 \cdot \frac{v}{r} \cdot T$

Is easy to prove that this total power is equal that the total power developed in a straight path at the same speed, so there are no losses of power when the vehicle makes a turn.

The basic system configuration for performing the ED with SFCs is shown in Figure 1.

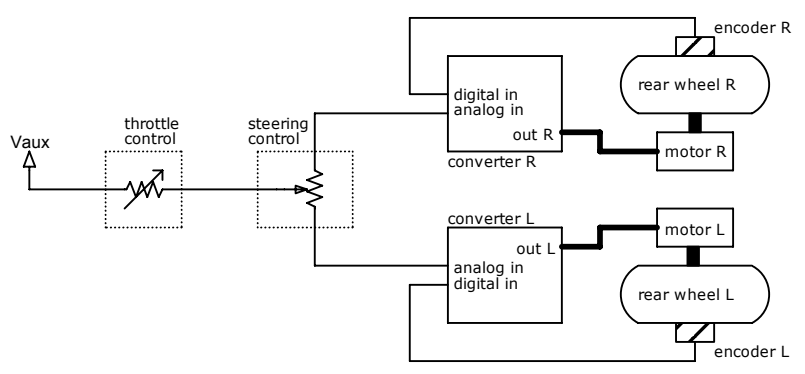

Fig. 1. Basic ED configuration

In addition to the throttle control, this configuration requires three sensors for proper operation: a steering angle sensor and a speed sensor at each wheel. The speed sensors are performed by digital encoders that are coupled with each traction wheel and are connected to a digital input of each converter. These sensors are needed to determine the actual frequency of motor rotation, because this frequency has a slip with respect to the frequency of the electric current supplied by the converter. This slip is a function of motor torque.

The steering angle sensor can be made by an analog potentiometer. This potentiometer and the the variable resistor that performs the throttle control are coupled and connected to an analog input of each converter. The resistor values are selected for verifying the Ackeraman equations. The analog input signals of the converters have the same value in a straight path and different values when cornering. Each converter performs a PID control with the analog input as reference signal and the digital input as feedback signal.

In order to minimize the cost and increase the reliability, it is beneficial the reduction of the number of sensors required for system operation. The digital encoders could be eliminated by using a useful function, the "slip compensation", which is usually implemented in SFCs. With this function, the converter can evaluate the actual rotating frequency of the motor as a function of the frequency of the electric current supplied by it, the nominal slip of the motor and the torque applied; therefore, the encoders are not necessary. The elimination of the feedback signal of the encoders also has the consequence that the control is simpler. This control comes down to programming the output frequency of each converter depending on the value of signals from throttle and steering angle.

The high performance of SFCs also permits that steering angle sensor can be eliminated. In this configuration, the input analog signal, coming from the throttle, has the same value in the two converters. The cost of this solution is a more complex control algorithm. The 
operation without a steering angle sensor is based on the torque of both engines must have the same value, regardless of whether the vehicle is moving straight ahead or taking a curve. SFCs can obtain an estimation of the instantaneous value of motor torque. Using this value as feedback signal, each converter can perform a PID control algorithm with the throttle value as reference signal. In this operating mode, the throttle does not directly controls the output frequency, but they controls the motor torque. Each converter regulates its output frequency for matching the motor torque with the torque reference, which is set by the throttle. Thus, when cornering, the output frequencies are optimally regulated for matching both motor torques, without the need to know the steering angle.

We have seen that the use of SFCs in association with $\mathrm{AC}$ induction motors enables to perform an ED in several ways. Other key feature of these systems in its application to vehicle traction is that they allow regenerative braking. Regenerative braking consists that the motor-drive system transforms the vehicle's kinetic energy into current to charge the batteries during deceleration. This function is inherently performed by the converters when they are supplied by a DC source (the batteries), connected directly to internal DC bus.

In the standard operation, SFCs are supplied by an AC source, normally the grid. The AC current is rectified in the input stage to obtain the DC bus voltage. During deceleration the motor works as a generator, providing power to the converter. This energy can't be returned to the grid, so the voltage of DC bus tends to increase. This increase can be dangerous for the system, so a braking resistor is necessary to dissipate excess energy. In the configuration of the SFC for vehicle traction, the DC bus is directly connected to the batteries, so that the regeneration current is used to recharge them and the braking resistor is not necessary.

To conclude this section, SFCs have other useful functions, such as detecting undertorque, which would allow to perform a more sophisticated traction control which suppresses tire slip when the vehicle is accelerating on an icy road, for example. The analysis and development of these functions is outside the scope of this paper.

\section{BEV design.}

To define the structure of the vehicle, we have imposed the condition that it is included within the category L6e (light quadricycles) according to European directive 2002/24/EC. We made this choice because such vehicles are primarily oriented to urban use or short distance routes, which are the environments where the $\mathrm{BEV}$ is competitive with ICV. following:

The main features of these category are the

[1] Maximum speed: $45 \mathrm{~km} / \mathrm{h}$.

[2] Maximum weight (excluding batteries): $350 \mathrm{~kg}$

[3] Maximum power: $4 \mathrm{KW}$
All other features necessary for the selection of the parameters of each component was taken as follows:

[4] Maximum slope at low speed: $8 \%$

[5] Maximum initial acceleration at flat route: 0,83 $\mathrm{ms}^{-2}$ (The increased speed is $15 \mathrm{~km} / \mathrm{h}$ in 5 seconds)

[6] Range (maximum distance traveled) in conditions of constant maximum speed and flat route: $75 \mathrm{~km}$.

From [3], we have selected two standard AC induction motors of 2,2 KW and $3000 \mathrm{rpm}$ at $50 \mathrm{~Hz}$ and two SFCs. The main rated characteristics of these motors are: torque: $T_{m}=7,5 \mathrm{~N} \cdot \mathrm{m}$; current: $I_{m}=4,6 \mathrm{~A}$; efficiency: $\eta_{m}=$ $83 \%$ and slip: $s=4,7 \%$. The average efficiency of each converter is $\eta_{c}=85 \%$

The road load has three components: rolling resistance $\left(f_{r o}\right)$, aerodynamic drag $\left(f_{l}\right)$, and climbing resistance $\left(f_{s t}\right)$ [1]. If we not consider the wind velocity, the loads are calculated as follows:

$$
\begin{aligned}
& f_{r o}=\left(0.012+0.0003 \cdot v^{1.1}\right) \cdot m \cdot g ; \\
& f_{l}=0.65 \cdot C_{X} \cdot A \cdot v^{2} ; \quad f_{s t}=m \cdot g \cdot \sin \alpha
\end{aligned}
$$

Where $m$ is the vehicle mass, $g$ is the gravitational acceleration constant, $C_{X}$ is the aerodynamic drag coefficient, $A$ is the vehicle frontal area, $v$ is the vehicle speed and $\alpha$ is the grade angle.

Assuming that maximum mass (with batteries and passengers) is $m=500 \mathrm{Kg} ; g=9.8 \mathrm{~ms}^{-2} ; C_{X}=0.3 ; A=1$ $\mathrm{m}^{2}$ and, taking into account (1) and (3), $v=12,5 \mathrm{~ms}^{-1}, \alpha=$ $0.08 \mathrm{rad}$ :

$$
f_{r o}=82.45 \mathrm{~N} ; f_{l \max }=30.47 \mathrm{~N} ; f_{s t}=392 \mathrm{~N}(1)
$$

If the velocity is low (as at starting), it can be removed from the above equations. With this assumption we obtain the following results:

- The force needed for accelerating the vehicle at flat route with zero initial speed is given by:

$F_{a} \simeq k_{m} \cdot m \cdot a_{\max }+f_{\text {ro }}$

Where $k_{m}$ is a coefficient that takes into account the rotating mass of the vehicle and $a_{\max }$ is the maximum acceleration (that is $0,83 \mathrm{~ms}^{-2}$ ). Assuming that $k_{m}=1$ : $F_{a}=475.47 \mathrm{~N}$

- The maximum force needed for climbing is:

$$
F_{c}=f_{r o}+f_{s t}=450.8 \mathrm{~N} \mathrm{(3)}
$$

Assuming that the wheel radius $(r)$ is $0.25 \mathrm{~m}$, and selecting the maximum value of (2) and (3), we can calculate the gear ratio $(G)$ :

$$
G=\frac{F_{a} \cdot r}{2 \cdot T_{m}}=7.92
$$

This is a non standard value. We have selected $G=7.5$

Finally, we have to calculate the size of the battery pack for reaching the value of range $(H)$ that is specified in [6]. 
The vehicle only has to overcome the road load, so if we consider the efficiency of motors, energy $(E)$ and electric charge (Q) consumption (assuming a DC bus of 216V) have the following values:

$$
E=\left(f_{r o}+f_{l}\right) \cdot \frac{H}{\eta_{m} \cdot \eta_{c}} ; \quad Q=\frac{E}{216}=15.44 \mathrm{Ah}
$$

Based on these calculations, we have made the final design of the vehicle:

- Welded steel chassis (total weight: 234 $\mathrm{Kg})$.

- $\quad$ Bodywork of moulded fiberglass (fig 2)

- Battery pack: 18 x NPC17-12 seriesconnected VRLA Yuasa batteries (fig 3)

- Power train: two 2,2 KW-3000 rpm standard AC induction motors (fig 3).

- Power controller: two VZAB2P2 Omron V1000 frequency converters. (fig 4).

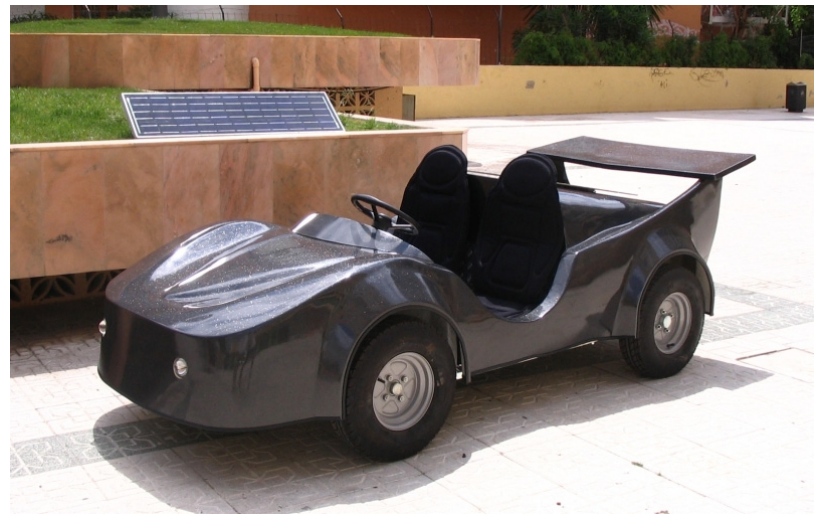

Fig. 2. General overview of the EV

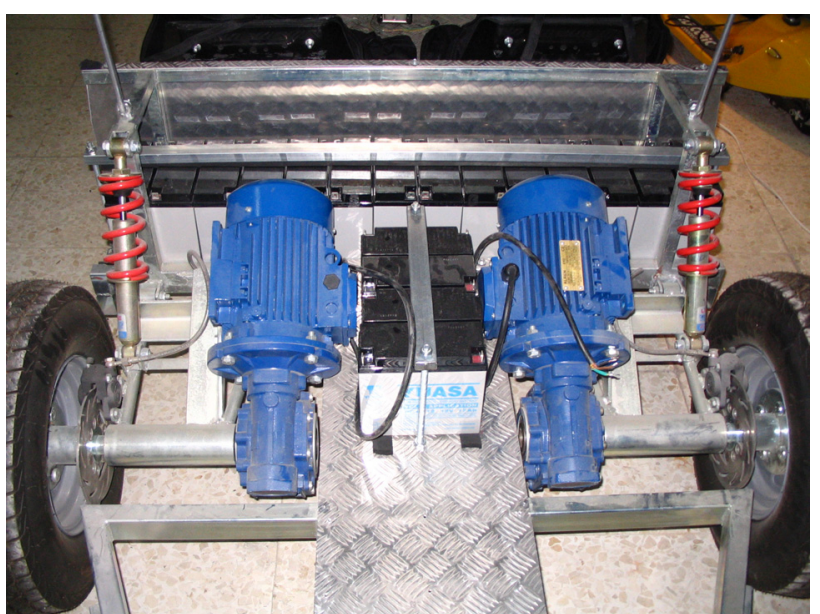

Fig. 3. Batteries and power train

\section{Conclusions}

We have designed and built an EV using standard industrial components whose main advantages are: simple structure, low cost, low maintenance and wide availability of components. Its main drawback is that its efficiency can be less than EVs based on dedicated components.

The development of such vehicles may be of interest to promote the technological independence of developing countries.

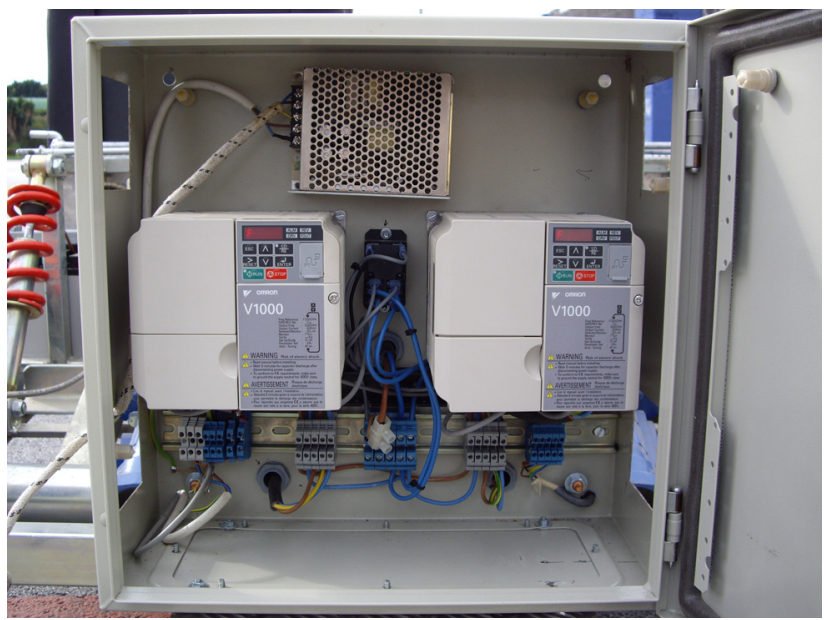

Fig. 4. Power controllers.

\section{References}

[1] J. Fullea García et al., El vehículo eléctrico: tecnología, desarrollo y perspectivas de futuro, Mc Graw Hill, Aravaca (1997)

[2] C. C. Chan, "The State of the Art of Electric and Hybrid Vehicles", Proceedings of the IEEE, Vol. 90, No. 2, (february 2002), pp. 245-275

[3] W. Kempton, J. Tomic, "Vehicle-to-grid power fundamentals: Calculating capacity and net revenue", Journal of power sources, Vol. 144, no. 1, (2005), pp. 268-279

[4] M. Alonso Abella, E. Lorenzo, F. Chenlo, "PV water pumping systems based on Standard Frequency Converters", Progress in Photovoltaics Research and applications. Vol. 11, No. 3, (2003), pp. 179-191

[5] L. Narvarte, E. Lorenzo and M. Aandam, "Lessons from a PV pumping programme in south Morocco", Progress in Photovoltaics Research and applications, Vol. 13, n. 3, (2005), pp. 261-270

[6] M. Ehsani, K. M. Rahman and H. A. Toliyat, " Propulsion System Design of Electric and Hybrid Vehicles", IEEE Transactions on industrial electronics, Vol. 44, No. 1, (february 1997), pp. 19-27

[7] S. Dhameja, Electric vehicle battery systems, Newnes, USA (2002), pp. 1-18

[8] F.J. Perez-Pinal, I. Cervantes and Ali Emadi, "Stability of an Electric Differential for Traction Applications", IEEE Transactions on Vehicular Technology, Vol. 58, No. 7, (September 2009), pp. 3224-3233 